\title{
Diabetes with heart failure increases methylglyoxal modifications in the sarcomere, which inhibit function
}

\author{
Maria Papadaki, ${ }^{1}$ Ronald J. Holewinski, ${ }^{2}$ Samantha Beck Previs, ${ }^{3}$ Thomas G. Martin, ${ }^{1}$ \\ Marisa J. Stachowski, ${ }^{1}$ Amy Li, ${ }^{3}$ Cheavar A. Blair, ${ }^{4}$ Christine S. Moravec, ${ }^{5}$ Jennifer E. Van Eyk, ${ }^{2}$ \\ Kenneth S. Campbell, ${ }^{4}$ David M. Warshaw, ${ }^{3}$ and Jonathan A. Kirk ${ }^{1}$ \\ 'Loyola University Chicago, Department of Cell and Molecular Physiology, Chicago, Illinois, USA. ${ }^{2}$ Cedars-Sinai Medical \\ Center, Heart Institute, Los Angeles, California, USA. ${ }^{3}$ University of Vermont, Department of Molecular Physiology and \\ Biophysics, Burlington, Vermont, USA. ${ }^{4}$ University of Kentucky, Department of Physiology, Lexington, Kentucky, USA. \\ ${ }^{5}$ The Cleveland Clinic, Department of Molecular Cardiology, Cleveland, Ohio, USA.
}

\begin{abstract}
Patients with diabetes are at significantly higher risk of developing heart failure. Increases in advanced glycation end products are a proposed pathophysiological link, but their impact and mechanism remain incompletely understood. Methylglyoxal (MG) is a glycolysis byproduct, elevated in diabetes, and modifies arginine and lysine residues. We show that left ventricular myofilament from patients with diabetes and heart failure (dbHF) exhibited increased MC modifications compared with nonfailing controls (NF) or heart failure patients without diabetes. In skinned NF human and mouse cardiomyocytes, acute MG treatment depressed both calcium sensitivity and maximal calcium-activated force in a dose-dependent manner. Importantly, dbHF myocytes were resistant to myofilament functional changes from MG treatment, indicating that myofilaments from dbHF patients already had depressed function arising from MG modifications. In human dbHF and MG-treated mice, mass spectrometry identified increased MG modifications on actin and myosin. Cosedimentation and in vitro motility assays indicate that MG modifications on actin and myosin independently depress calcium sensitivity, and mechanistically, the functional consequence requires actin/myosin interaction with thin-filament regulatory proteins. MC modification of the myofilament may represent a critical mechanism by which diabetes induces heart failure, as well as a therapeutic target to avoid the development of or ameliorate heart failure in these patients.
\end{abstract}

Authorship note: MP and RJH contributed equally to this work.

Conflict of interest: The authors have declared that no conflict of interest exists.

License: Copyright 2018, American Society for Clinical Investigation.

Submitted: March 22, 2018

Accepted: September 4, 2018 Published: October 18, 2018

\section{Reference information:} JCI Insight. 2018;3(20):e121264. https://doi.org/10.1172/jci. insight.121264.

\section{Introduction}

In patients with diabetes mellitus, which affects almost $10 \%$ of Americans, the primary causes of death are cardiovascular disorders $(1,2)$. The Framingham Heart Study found that diabetes led to a 5-fold (in women) and 2.4-fold (in men) higher incidence of heart failure compared with age-matched controls, even when controlling for age, blood pressure, weight, and coronary heart disease (3). However, the pathophysiological mechanisms by which diabetes can lead to heart failure are incompletely understood. As the number of people affected by diabetes increases worldwide, discovery of new molecular abnormalities that promote heart failure in diabetics is necessary to provide possible therapeutic targets.

In diabetes, hyperglycemia results in an increase in advanced glycation end products (AGEs) that modify extracellular matrix proteins and bind to receptors of AGE (RAGEs) that can induce maladaptive intracellular signaling (4). Plasma levels of AGEs predict heart failure hospitalization and mortality in diabetic patients (5). One of these glycating agents is an $\alpha$-oxoaldehyde called methylglyoxal (MG), a glycolysis byproduct, although lipid and protein metabolism pathways also contribute. While MG has physiological roles $(6,7)$, it is a reactive carbonyl species that can be toxic to the cell because it induces posttranslational modifications (PTMs) through an irreversible nonenzymatic reaction with unprotonated arginine and lysine residues. The primary MG-induced PTMs (MG-modifications) are arginine-derived hydroimidazolone N8-(5-hydro-5-methyl-4-imidazolon-2-yl)-ornithine (MG-H1) and lysine-derived Ne-carboxy-ethyl-lysine (CEL), although additional adducts may also be formed (8). Thus, MG is kept at low levels through its 
metabolism to D-lactate via the glyoxalase pathway (9). In patients with diabetes, MG levels are increased due to hyperglycemia, elevated ROS, and a reduction in the glyoxalase pathway (10).

The prevailing understanding of the pathophysiological mechanisms of AGEs and MG in diabetes primarily involve extracellular mechanisms (11-13) and receptor-based signaling that recognizes broadly modified proteins such as albumin $(14,15)$. However, MG can cross the plasma membrane as well as be generated intracellularly, and recent evidence shows it can induce modifications on several proteins in the cardiomyocyte to directly alter function. For example, MG can modify ryanodine receptor 2 and SERCA2a to disrupt normal myocyte calcium handling (16-19). Myosin heavy chain (MHC) has also been shown to be carbonylated (although not necessarily by MG) in a rat model of diabetes (20). While these studies indicate that intracellular MG-modifications can alter function, they do not directly address the human pathophysiology of diabetes and whether these modifications have a functional impact in the human disease.

Previous studies have shown that diabetes in humans (21) and experimental models $(22,23)$ reduces myofilament calcium sensitivity and possibly maximal calcium-activated force, suggesting myofilament dysfunction could underlie the depressed contractile function in human diabetes with heart failure (dbHF). However, the observed mechanisms, MHC isoform shifting and cTnI phosphorylation, are associated with general cardiomyopathy (24), and are thus likely to be a consequence of the associated cardiomyopathy and not an underlying mechanism by which diabetes induces heart failure. Therefore, the mechanistic link between diabetes and myofilament dysfunction is still unknown. We hypothesized that in human diabetes, MG induces specific intracellular modifications on myofilament proteins leading to impaired myofilament function.

We used left ventricular (LV) tissue samples from hearts explanted from dbHF patients, organ donors who did not have diabetes or heart failure, and mouse LV myocytes treated with varying concentrations of MG to address this hypothesis. We used antibody-based and mass spectrometry-based (MS-based) approaches to determine the extent to which overall MG-modifications are altered as well as to discover the individual residues that are modified in human diabetes and with direct exposure to MG. Force-calcium relationships, myofilament protein cosedimentation, and in vitro motility assays were used to identify the functional consequence of these modifications and the mechanisms involved. While clinical trials to reduce AGEs have been unsuccessful (25), the myofilament is a highly tunable functional system that can be augmented therapeutically through a toolbox of small molecules (26). The results from this study may suggest a new treatment target to improve function in cardiovascular diseases due to diabetes or to prevent their development.

\section{Results}

Diabetes with heart failure increases myofilament MG-modifications. Patient characteristics for the nonfailing controls (NF), dbHF, and dilated cardiomyopathy patients without diabetes (DCM) are shown in Table 1. MG levels were measured in myofilament-enriched fractions from NF, dbHF, and DCM human heart LV tissue. The dot blot antibody-based approach showed a significant increase in overall MG-modifications in dbHF compared with NF controls and DCM (MG/tubulin normalized to NF, NF = $1.00 \pm 0.04, n=4 ; \mathrm{dbHF}=$ $1.45 \pm 0.15, n=6 ; \mathrm{DCM}=1.07 \pm 0.05, n=4 ; P=0.006$; Figure $1, \mathrm{~A}$ and $\mathrm{B}$ ). The antibody only recognizes MG-H1 modifications, and not CEL modifications or others. This is a limitation of the approach, although MG-H1 modifications have been shown to be the most abundant MG-modification (27, 28).

MS analysis also showed that myofilament-enriched samples from dbHF patients had an increased quantity of MG-modifications compared with NF (MS/MS spectral counts, NF = 33.8 $\pm 2.6, n=4 ; \mathrm{dbHF}=47.2 \pm$ $2.2, n=5, P=0.0049$; Figure 1C). There was high quantitative agreement between the antibody- and MS-based approaches ( $45 \%$ and $40 \%$ increase in $\mathrm{dbHF}$ versus $\mathrm{NF}$, respectively). In total, $32 \mathrm{MG}$-modifications were identified in human myofilament-enriched LV samples: 9 that were only identified in the dbHF patient samples, 0 that were only detected in NF, and 23 that were identified in both NF and dbHF (Figure 1D).

$M G$ decreases myofilament function in mouse skinned myocytes. To determine the functional effect of MG-modifications on the cardiac myofilament, force-calcium relationships were measured in isolated LV skinned myocytes from male, 3- to 4-month-old, C57BL/6J mice, before and after a 20-minute exposure to MG. In the skinned myocyte experiment the cell is chemically permeabilized, and the myocyte is exogenously exposed to calcium, ATP, and creatine kinase. Therefore, the assay readout is specific to myofilament function. Posttreatment function was measured with zero MG in the bathing solutions, so the functional consequences arise only from the modifications induced during the exposure period. Average force-calcium curves before and after treatment with $10 \mu \mathrm{M}$ and $100 \mu \mathrm{M}$ MG are shown in Figure 2, A and B. Acute exposure to $10 \mu \mathrm{M}$ MG caused a statistically significant decrease in calcium sensitivity, indicated by 
Table 1. Patient characteristics

\begin{tabular}{|c|c|c|c|c|}
\hline Characteristic & NF $(n=7)$ & dbHF $(n=6)$ & $\operatorname{DCM}(n=4)$ & $P$ value NF vs. dbHF \\
\hline Age & $51 \pm 7$ & $60 \pm 3$ & $58 \pm 3$ & 0.28 \\
\hline$\%$ Female & $57 \%$ & $67 \%$ & $50 \%$ & 1 \\
\hline \% Type II Diabetes & $0 \%$ & $100 \%$ & $0 \%$ & $<0.001$ \\
\hline$\%$ Ischemia & $0 \%$ & $67 \%$ & $0 \%$ & 0.021 \\
\hline$\%$ Myocardial Infarction & $0 \%$ & $33 \%$ & $0 \%$ & 0.19 \\
\hline$\%$ Diastolic Dysfunction & $0 \%$ & $83 \%$ & $?$ & 0.005 \\
\hline
\end{tabular}

$\mathrm{NF}$, nonfailing donor heart rejected for transplant; dbHF, patients with diabetes with heart failure; DCM, patients with dilated cardiomyopathy; LVEF, left ventricular ejection fraction as measured by echocardiography; ?, full clinical data not available.

an increase in $\mathrm{EC}_{50}$ (pretreatment $=1.46 \pm 0.23 \mu \mathrm{M}$, posttreatment $=2.29 \pm 0.32 \mu \mathrm{M}, n=9$ myocytes from 3 different mice, $P=0.006$; Figure 2C). When the concentration of $\mathrm{MG}$ was increased to $100 \mu \mathrm{M}$, the calcium sensitivity was also depressed (pretreatment $=1.86 \pm 0.18 \mu \mathrm{M}$, posttreatment $=2.72 \pm 0.44 \mu \mathrm{M}, n=$ 9 myocytes from 3 mice, $P=0.01$ ) (Figure 2D). The corresponding changes in maximal calcium-activated force $\left(\mathrm{F}_{\max }\right)$, however, did show differences between these two concentrations of MG. While $10 \mu \mathrm{M}$ MG had no effect on maximal calcium-activated force $(P=0.33$, Figure $2 \mathrm{C})$, there was a significant depression when the myofilament was exposed to the higher $100 \mu \mathrm{M}$ dose of $\mathrm{MG}$ (pretreatment $=26.4 \pm 2.8 \mathrm{mN} /$ $\mathrm{mm}^{2}$; posttreatment $=22.3 \pm 1.9 \mathrm{mN} / \mathrm{mm}^{2}, n=9$ myocytes from 4 mice, $P=0.02$; Figure $2 \mathrm{D}$ ). Two-way repeated-measures ANOVA indicated an increasing dose-response for both $\mathrm{F}_{\max }$ and $\mathrm{EC}_{50}(P=0.032$ and $P<0.001$, respectively; Figure 2, E and F). With $1 \mu \mathrm{M} \mathrm{MG}$, there was no effect on $\mathrm{F}_{\max }$, although there was a trend towards a decrease in calcium sensitivity. As expected, there was no effect on any functional measurement after 20-minute incubation with 0 MG (relax solution only).

The addition of the MG dicarbonyl adducts to arginine and lysine residues is irreversible, so if the sarcomeric functional consequences are due to these modifications, the changes in $\mathrm{F}_{\max }$ and $\mathrm{EC}_{50}$ should also be irreversible. To test this, myocytes were preincubated in $100 \mu \mathrm{M} \mathrm{MG}$ for 20 minutes, a force-calcium relationship measured, the MG was washed out for 20 minutes, and then a second force-calcium relationship was measured. Not only were the effects on $\mathrm{F}_{\max }$ and $\mathrm{EC}_{50}$ not reversed, there was a further decrease in calcium sensitivity over the washout period (Figure 3 ).

Altered calcium sensitivity arises primarily from changes in phosphorylation levels of thin filament proteins. To confirm that MG was not interacting with and altering phosphorylation through an unknown crosstalk mechanism, we used a phosphoprotein and total protein gel stain on samples prepared from skinned myocytes before and after 20-minute exposure to MG (same conditions as functional experiments). Supplemental Figure 1 (supplemental material available online with this article; https://doi.org/10.1172/jci. insight.121264DS1) shows that there was no change in myofilament protein phosphorylation. Likewise, diastolic dysfunction is a common functional consequence of dbHF (29), and can arise from titin-based stiffness that results in higher single myocyte passive stiffness (30). With 20-minute exposure to $100 \mu \mathrm{M} \mathrm{MG}$, we found no effect on passive stiffness (Supplemental Figure 2). This lack of effect is supported by recent data suggesting the diabetes-induced increase in passive stiffness occurs because of altered cGMP/PKG signaling (31).

Functional role of MG-modifications in $\mathrm{dbHF}$ and NF human myocytes. After determining that myofilament MG-modifications increase in human $\mathrm{dbHF}$ and that these depress myofilament function in mouse myocytes, we aimed to determine the functional consequence of these modifications in $\mathrm{dbHF}$ patients. There is no known method for reversing these modifications, and so they cannot be removed in dbHF hearts to observe a restoration of function. For this reason, our approach was to treat skinned myocytes with increasing doses of MG with the expectation that there would be a differential response between NF and $\mathrm{dbHF}$ if there was a baseline functional difference due to existing MG-modifications induced by diabetes. When human LV skinned myocytes were treated with $10 \mu \mathrm{M}$ MG for 20 minutes, NF and dbHF myocytes experienced similar decreases in calcium sensitivity $\left(\Delta \mathrm{EC}_{50}: \mathrm{NF}=0.94 \pm 0.21 \mu \mathrm{M}, n=8, P=0.020\right.$; 
A

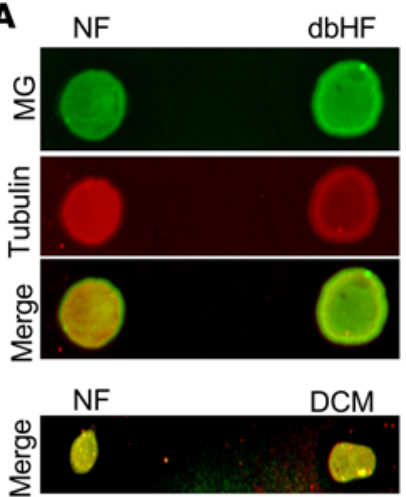

\section{C}

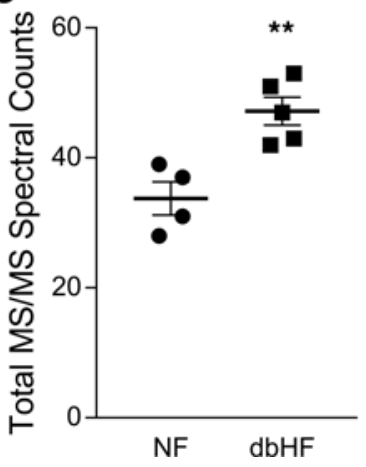

B

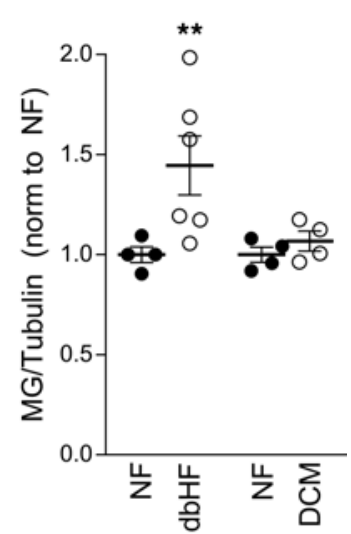

D

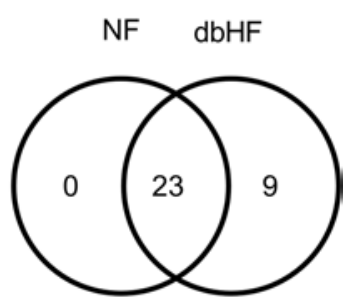

Figure 1. Methylglyoxal-modifications are elevated in the myofilament of human diabetic patients with heart failure. (A) Representative 2-colored dot blot showing methylglyoxal (MG, green), $\alpha$-tubulin (red), and merge of the myofilament fraction of nonfailing control (NF) samples or diabetes with heart failure samples (dbHF). Below is shown the merge channel for MG (green) and $\alpha$-tubulin (red) for NF and dilated cardiomyopathy (DCM) left ventricular myofilament samples. (B) Quantification of the MG/tubulin ratio in myofilament fraction of NF ( $n=$ 4) versus dbHF $(n=6)$ and NF $(n=4)$ versus DCM $(n=4)$ samples. ${ }^{* *} P<0.01$ using 2-way ANOVA for disease and blot. (C) Tandem mass spectrometry (MS/MS) spectral counts for total MG-modifications for either NF (black dots) $(n=4)$ or dbHF (black squares) $(n=5)$ myofilament samples. ${ }^{*} P<0.01$, using unpaired $t$ test. (D) Venn diagram of MG-modifications detected in NF and dbHF samples by MS.

$\mathrm{dbHF}=1.09 \pm 0.35 \mu \mathrm{M}, n=8, P=0.022)$ and maximal calcium activated force $\left(\Delta \mathrm{F}_{\text {max }}: \mathrm{NF}=-10.04 \pm 2.55\right.$ $\mathrm{mN} / \mathrm{mm}^{2}, P=0.004 ; \mathrm{dbHF}=-7.40 \pm 1.90 \mathrm{mN} / \mathrm{mm}^{2}, P=0.011 ;$ Figure 4).

With $100 \mu \mathrm{M}$ MG, NF and dbHF cardiomyocytes showed no further decrease in $\mathrm{F}_{\max }$ compared with $10 \mu \mathrm{M} \mathrm{MG}\left(\Delta \mathrm{F}_{\max }: \mathrm{NF}=-6.46 \pm 1.81 \mathrm{mN} / \mathrm{mm}^{2}, n=7, P=0.011 ; \mathrm{dbHF}=-5.11 \pm 1.5 \mathrm{mN} / \mathrm{mm}^{2}, n=\right.$ $6, P=0.020)$. However, the skinned myocytes from dbHF myocytes saw no additional change in calcium sensitivity compared with $10 \mu \mathrm{M}$, in contrast to the skinned myocytes from NF controls $\left(\triangle \mathrm{EC}_{50}: \mathrm{NF}=2.56\right.$ $\pm 0.57, P=0.002 ; \mathrm{dbHF}=1.06 \pm 0.32, P=0.014$; difference between $\mathrm{NF}$ and $\mathrm{dbHF} P<0.001$ using 2 -way repeated-measures ANOVA; Figure 4).

Specific MG-modifications on actin and myosin. MS was used to identify specific sites that were quantitatively altered in human dbHF patients versus NF controls and those that were induced by acute MG exposure in the mouse. Skinned myocytes were prepared using the same methods as for the functional studies. Since trypsin cleaves at arginine and lysine, the same residues that are modified by MG, 2 protocols were used to digest the samples into peptides. The first used a long exposure time to ensure that the samples were fully digested in the event that MG-modifications alter the efficacy of trypsin digestion. The second protocol used a short exposure to avoid fragmenting the modifications, as has been suggested previously (32).

For the mouse samples, we first analyzed the effect of $100 \mu \mathrm{M}$ MG exposure for 20 minutes, the same as the functional studies. However, we were unable to detect any statistically significant quantitative changes in mouse skinned myocytes before and after this treatment (pretreatment $=37.2 \pm 5.0$ spectral counts per biological sample, posttreatment $=32.5 \pm 1.0$ spectral counts, $n=6, P>0.05$ ). Unfortunately, like most PTMs, MG-modifications occur at a relatively low stoichiometry and are thus challenging to detect via MS. While enrichment techniques exist for many PTMs to increase the likelihood of their detection, no such techniques exist for MG-modifications. To overcome this issue, we increased our detection ability by MS by treating with $100 \mathrm{mM}$ MG for 20 minutes (pre-enrichment). This dose of MG allowed us to detect a significant increase in total MG-modifications (pretreatment $=32.0 \pm 4.0$ spectral counts, posttreatment $=79.9 \pm 14.7$ spectral counts, $n=4, P=0.02$ )

In both the mouse and the human samples, several residues on actin and myosin exhibited increased MG-modifications (Table 2) that we hypothesize are responsible for the functional effects MG exerts on cardiac contractility. Specifically, with the long trypsin digestion, MG-modification of K384 on $\alpha$-MHC was found 
A
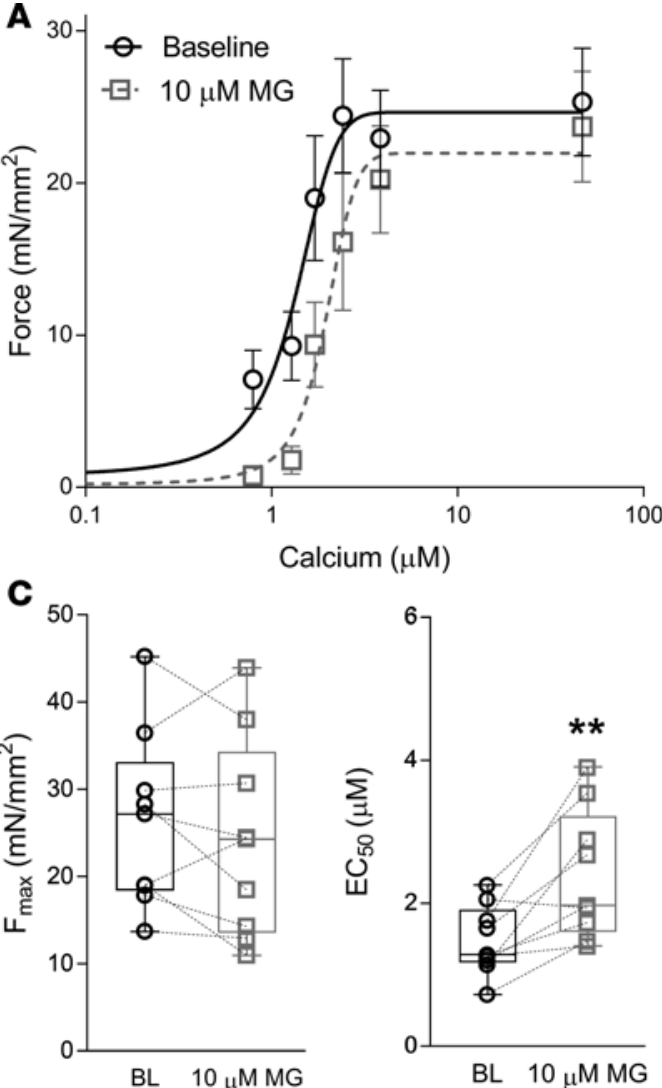

$\mathrm{BL} \quad 10 \mu \mathrm{MMG}$

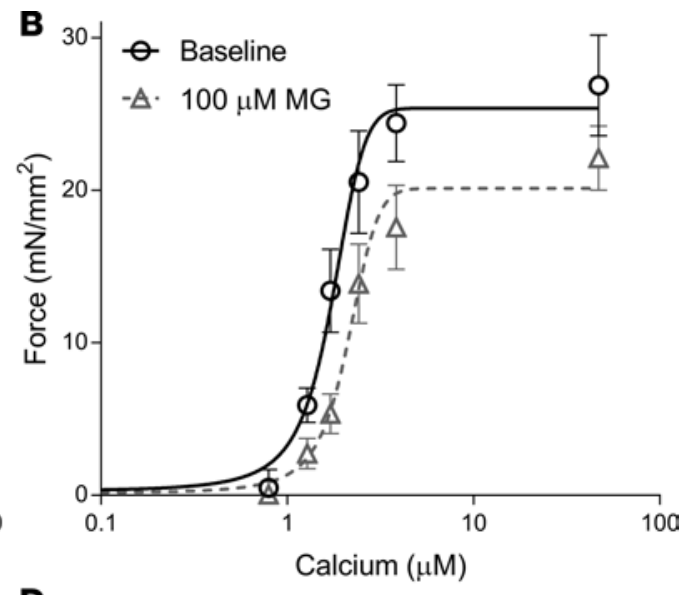

D
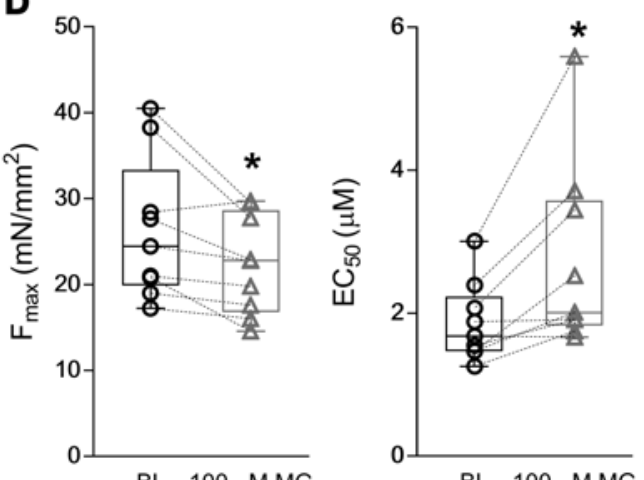

$\mathbf{F}$

- Before Tx - After Tx
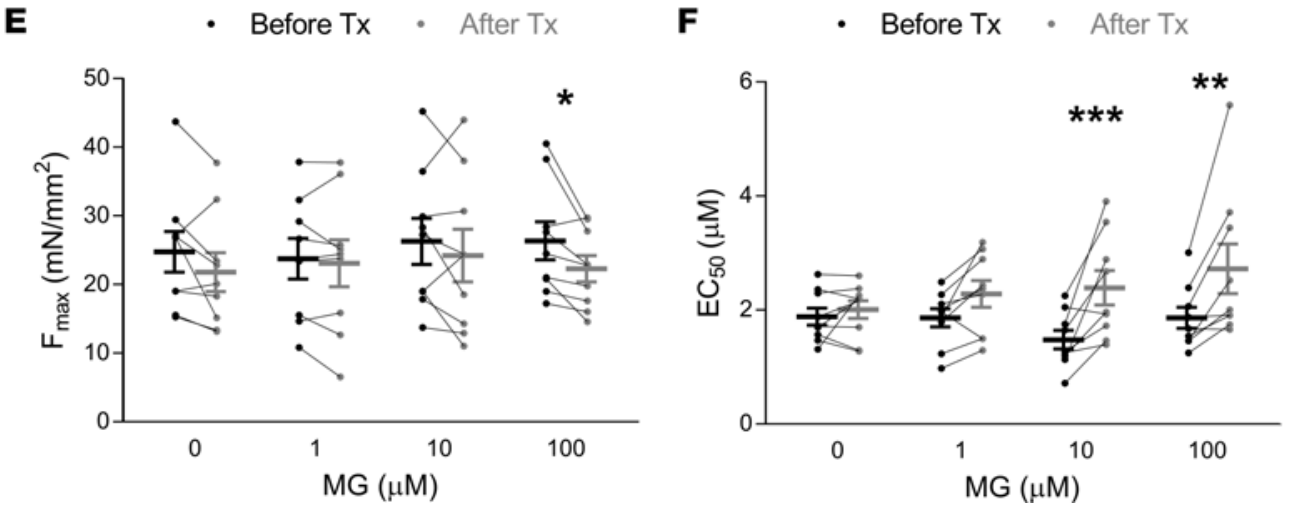

Figure 2. Effect of methylglyoxal on mouse skinned myocyte function. (A) Mean force as a function of calcium concentration and fitted curves for skinned myocytes before (open circles, black line) and after (open gray squares, gray dotted line) treatment with $10 \mu \mathrm{M}$ methylglyoxal (MC) for 20 minutes ( $n=9$ per group). (B) Mean force as a function of calcium concentration and fitted curves for myocytes before (open circles, black line) and after (open gray squares, gray dotted line) treatment with $100 \mu \mathrm{M}$ MG for 20 minutes ( $n=9$ per group). (C) All calculated values for individual myocytes and summary box plot before (black) and after (gray) $10 \mu \mathrm{M} \mathrm{MC}$ on maximal calcium-activated force $\left(\mathrm{F}_{\text {max }}\right.$, left) and $\mathrm{Ca}^{2+}$ sensitivity ( $\mathrm{EC}_{50}$, right). Individual myocytes before and after treatment are connected by dotted lines. (D) All individual calculated values and summary box plot showing the effect of $100 \mu \mathrm{M} \mathrm{MG}$ on $\mathrm{F}_{\max }$ and $\mathrm{EC}_{50^{\circ}}$. (E) Dose effect of 1-100 $\mu \mathrm{M}$ $\mathrm{MG}$ on $\mathrm{F}_{\max }$ and $(\mathbf{F}) \mathrm{EC}_{50}$ of skinned mouse myocytes. ${ }^{*} P<0.05$, ${ }^{* *} P<0.01,{ }^{* *} P<0.001$ by paired $t$ test $(\mathbf{C}$ and $\mathbf{D})$ or 2-way repeated-measures ANOVA (E and $\mathbf{F}$ ).

significantly increased in dbHF compared with $\mathrm{NF}$, and R370 on the same protein also trended towards an increase. Using short trypsin digestion, we further discovered significant increases in MG-modifications on actin K70 and K1899 on embryonic skeletal MHC. Two MG-modifications, actin K70 and $\alpha$-MHC R370, were also detected in the mouse MG-treated samples. In the mouse, additional MG-modifications were identified after MG treatment compared with before treatment, including actin K293, $\alpha-\mathrm{MHC}$ K944, and essential light chain R40, R103, and K180 (Table 2). Representative spectra for several of these modifications are shown in Figure 5. 

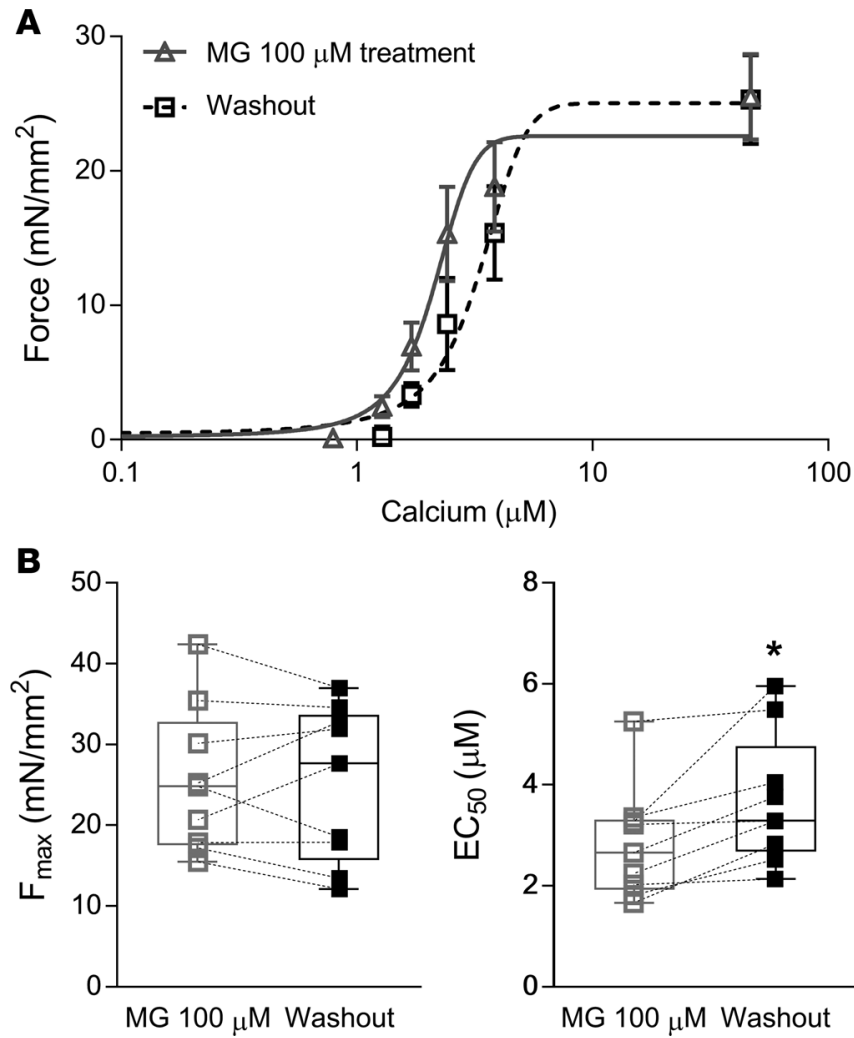

Figure 3. Reversibility of the effect of MG on skinned myocytes. (A) Mean and SEM of force as a function of calcium concentration and fitted curves for skinned myocytes pretreated with 100 $\mu \mathrm{M}$ methylglyoxal (MG) for 20 minutes (open triangles, gray line) and then incubated with relaxing solution for 20 minutes (open squares, black dotted line). (B) All $F_{\text {max }}$ (left) and $\mathrm{EC}_{50}$ (right) data and summary box plots for myocytes pretreated with $100 \mu \mathrm{M}$ MG for 20 minutes (gray open squares) and then incubated with relaxing solution for 20 minutes (black squares). Individual myocytes with paired data before and after washout are connected by dotted lines $(n=9) .{ }^{*} P<0.05$ using paired $t$ test.

The MG-modified sites identified were primarily on actin and myosin. It is possible that MS was oversampling these two highly abundant proteins and missed MG-modifications on other myofilament proteins. Furthermore, the functional impact we observed on calcium sensitivity suggested possible alterations to thin filament-associated proteins. To address this, we ran human NF $(n=4)$ and dbHF $(n=5)$ myofilament-enriched samples on an SDS-PAGE gel, silver stained, and excised the bands corresponding to tropomyosin, troponin T, troponin C, troponin I, and actin (as a positive control, since we already observed modified peptides on actin). Each of these bands ( 5 bands for each of the 9 hearts) were then independently digested and examined via MS. Despite good protein coverage for tropomyosin (70\% coverage), troponin T (45\%), troponin C (25\%), and troponin I (55\%), no MG-modifications were detected on any of these proteins in either NF or dbHF samples (Supplemental Figure 3 ). There was $67 \%$ sequence coverage for actin, and in agreement with our previous analysis, several MG-modifications were detected.

Actin and myosin modifications independently depress thin filament regulation. The MS data suggest that the decrease in myofilament function is due to MG-modifications on actin and/or myosin. We first aimed to determine whether these modifications altered actin-myosin interaction directly using a cosedimentation assay. Using bare actin and myosin, 20-minute treatment with $100 \mu \mathrm{M}$ MG had no effect on actin-myosin binding (Figure 6A). However, when the assay was performed with regulated actin (i.e., native thin filaments, NTFs, which include actin, tropomyosin, troponin I, troponin C, and troponin T) at $0.145 \mu \mathrm{M}$ calcium, MG reduced NTF-myosin binding as measured by cosedimentation (Figure $6 \mathrm{~B}, n=3, P=0.022$ for MG treatment, $P=0.017$ for interaction by 2-way repeated-measures ANOVA). These data suggest that the functional effects of MG-modifications are apparent only in calcium-regulated systems such as myosin binding to NTFs or in the skinned myocyte.

Cosedimentation can quantify the amount of protein bound to filamentous actin but does not necessarily correlate to any mechanical indices of function in the skinned myocyte experiments. We therefore examined the functional effects of MG exposure at the molecular level using an in vitro motility assay. In this assay, NTFs slide along a bed of immobilized cardiac myosin in a calcium-dependent manner, with the fraction of moving NTFs taken as an indicator of NTF activation state (33). By exposing myosin or NTFs to various doses of MG and then characterizing the fraction of NTFs moving with these modified 

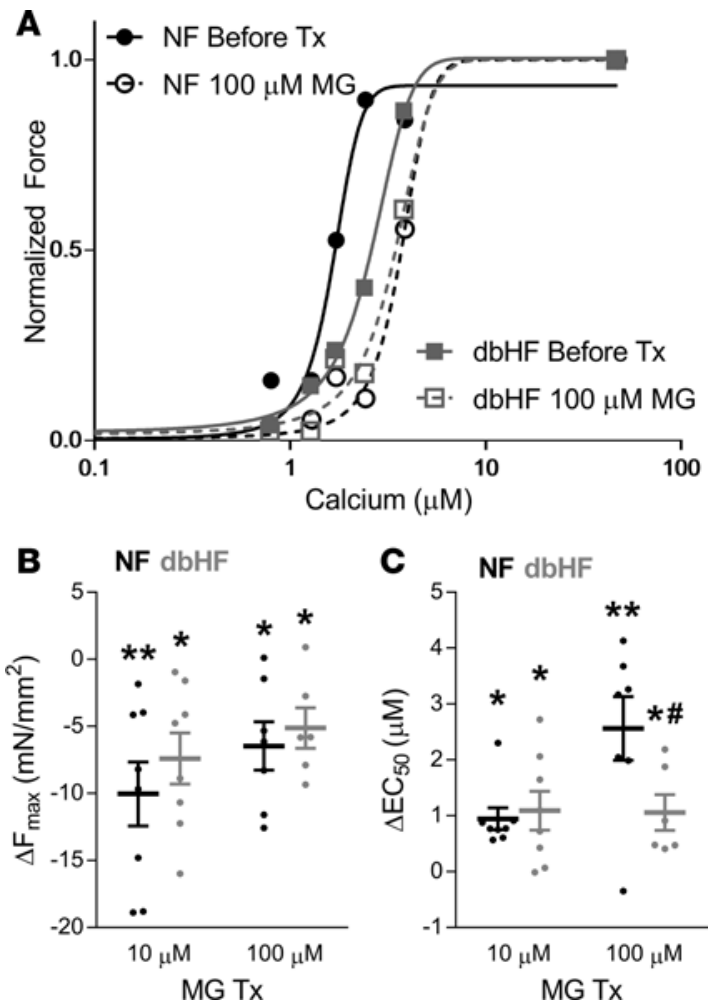

Figure 4. Effect of methylglyoxal on human dbHF and NF skinned myocytes. (A) Representative force normalized to $F_{\max }$ as a function of calcium concentration and fitted curves for a skinned cardiomyocyte from nonfailing (NF) human before (black circles, black solid line) and after treatment with $100 \mu \mathrm{M}$ methylglyoxal (MG) (black open circles, black dotted line) as well as a diabetic heart failure (dbHF) cardiomyocyte before (gray squares, gray solid line) or after treatment with $10 \mu \mathrm{M}$ MG (gray open squares, gray dotted line). (B) Change in $F_{\text {max }}$ $\left(\Delta \mathrm{F}_{\max }=\right.$ posttreatment - pretreatment $)$ for $\mathrm{NF}$ and $\mathrm{dbHF}$ treated with $10 \mu \mathrm{M} \mathrm{MG}(\mathrm{n}: \mathrm{dbHF}=8, \mathrm{NF}=8)$ or $100 \mu \mathrm{M} \mathrm{MG}(n: \mathrm{dbHF}=6, \mathrm{NF}=7)$ shown as the mean \pm SEM. (C) Change in $\mathrm{EC}_{50}$ for $\mathrm{NF}$ and dbHF treated with $10 \mu \mathrm{M} \mathrm{MC}(n: \mathrm{dbHF}=8, \mathrm{NF}=8)$ or 100 $\mu \mathrm{M} \mathrm{MG}(n: \mathrm{dbHF}=6, \mathrm{NF}=7)$ shown as the mean \pm SEM. ${ }^{*} P<0.05$ pretreatment versus posttreatment, ${ }^{*} P<0.01$ pretreatment versus posttreatment; ${ }^{\#} P<0.001 \mathrm{dbHF}$ versus $\mathrm{NF} \Delta \mathrm{EC}_{50}$ by 2 -way repeated-measures ANOVA and Holm-Sidak post hoc test. Tx, treatment.

proteins, we could differentially determine whether MG-modifications to myosin or actin were responsible for any molecular alterations in NTF calcium sensitivity. We first showed that the NTFs were calcium regulated as indicated by an increase in fraction of moving filaments as a function of calcium (Figure 6C, $n=4)$, while additional experiments following MG exposure were carried out at $0.175 \mu \mathrm{M} \mathrm{Ca}^{2+}$, near the average $\mathrm{EC}_{50}$ (33). When only MG-exposed myosin, MG-exposed NTFs, or both MG-exposed myosin and NTFs were used in the assay, there was a dose-dependent decrease in the fraction moving (i.e., reduced calcium sensitivity; Figure $6, \mathrm{D}$ and $\mathrm{E}, n=5$ per group, $P=0.042$ for MG treatment by 2-way ANOVA). However, there was no difference between the component treated (myosin vs. NTFs vs. both, $P=0.912$ for component, $P=0.99$ for interaction between MG dose and component treated). The apparent noise in the NTF activation state (i.e., fraction moving) (Figure 6E) most likely stems from the chosen calcium level of $0.175 \mu \mathrm{M} \mathrm{Ca}^{2+}$ ( $\mathrm{pCa}$ 6.75) being on the steepest part of the fraction moving versus $\left[\mathrm{Ca}^{2+}\right]$ relationship (Figure 6C). Thus, MG's functional effects require a regulated thin filament system (actin and tropomyosin/troponin complex) and are mediated independently through either actin or myosin modifications with no additive effect from modifications on both. However, the MG-modifications themselves are not calcium dependent, since in the cosedimentation and in vitro motility assays the proteins are exposed to the MG before they are activated by calcium.

\section{Discussion}

Diabetes results in a significant increase in MG (34), a dicarbonyl reactive carbonyl species that reacts with arginine and lysine residues to induce irreversible PTMs. We report for the first time to our knowledge that human heart tissue explanted from dbHF patients exhibited an increase in total myofilament MG-modifications compared with NF human hearts, while no change was observed in DCM patients. Furthermore, acute exposure to MG depressed myofilament contractile function in both mouse and human myocytes. MS analysis of human dbHF and MG-treated mouse cardiac myofilament identified what we believe are novel modifications on myofilament proteins, specifically actin and myosin, but not tropomyosin or the troponin complex (thin filament regulatory proteins). However, the MG-modifications on actin and myosin required the presence of a calcium-regulated NTF with its tropomyosin and troponin complex to realize the functional effects in the in vitro motility assay. Interestingly, modifications on either actin or myosin depress NTF calcium sensitivity, but these effects were not additive when both MG-treated myofilaments 
Table 2. MG-modifications on myofilament fraction found in human and mouse

\begin{tabular}{|c|c|c|c|c|c|c|c|c|}
\hline & & & & Human & & & Mouse & \\
\hline Actin & ACTC & K70 & $9.5 \pm 1.3$ & $13.7 \pm 1.1$ & 0.038 & $10.3 \pm 1.4$ & $10.3 \pm 1.5$ & 0.99 \\
\hline$\alpha-\mathrm{MHC}$ & MYHG & R370 & $0.8 \pm 0.3$ & $2.2 \pm 0.6$ & 0.11 & $0 \pm 0$ & $1.3 \pm 0.5$ & 0.04 \\
\hline$\alpha-\mathrm{MHC}$ & MYHG & K384 & $5.3 \pm 0.9$ & $9.4 \pm 1.0$ & 0.02 & N.D. & N.D. & - \\
\hline$\alpha-\mathrm{MHC}$ & MYHG & K944 & N.D. & N.D. & - & $0 \pm 0$ & $2.8 \pm 0.6$ & 0.005 \\
\hline ELC & MYL3 & R103 & N.D. & N.D. & - & $0 \pm 0$ & $4.3 \pm 0.8$ & 0.001 \\
\hline ELC & MYL3 & K180 & N.D. & N.D. & - & $0 \pm 0$ & $2.8 \pm 0.8$ & 0.01 \\
\hline
\end{tabular}

$\mathrm{NF}$, nonfailing controls; dbHF, diabetes and heart failure; BL, baseline; ELC, myosin essential light chain; MC Tx, treatment with 100 mM MC for 20 minutes; N.D. not detected. Values represent mean MS/MS spectral counts $\pm \mathrm{SEM}$ ( $n: \mathrm{NF}=4, \mathrm{dbHF}=5 ; n=6$ for mouse BL and MG Tx). $P$ values were calculated using an unpaired $t$ test for the human data and a paired $t$ test for the mouse data.

were combined in the motility assay. Consistent with a functional deficit caused by these modifications in human dbHF, myocytes from these patients had a significantly reduced functional response to MG exposure, compared with NF controls. These findings suggest a novel target to treat symptoms of heart failure due to diabetes or to avoid its development in diabetic patients.

The myofilament contractile system is highly cooperative, where small changes in protein modifications $(<10 \%)$ can have dramatic functional affects $(35,36)$. Therefore, it is not surprising that a $40 \%-45 \%$ increase in total MG-modifications, as shown by antibody- and MS-based methods, resulted in a significant functional impact. It was surprising that $\mathrm{MG}$ depressed both calcium sensitivity and $\mathrm{F}_{\max }$, as most factors affect either one or the other but rarely both. Interestingly, we found that mouse myocytes showed no change in $\mathrm{F}_{\max }$ upon treatment with $10 \mu \mathrm{M} \mathrm{MG}$, while human myocytes did. The reason for that difference might be the dissimilar myosin isoforms present in human and mouse cardiac muscle that might be affected by MG-modifications in distinct functional ways.

While many studies quantify protein AGEs, comparatively few have quantified specific modified residues (37), and fewer still also characterize a functional consequence as we have done here. Considering both our functional and site-specific data, it seemed likely that MG would directly inhibit actin-myosin interactions (38). Cosedimentation analysis using MG-treated bare actin and myosin contradicted this hypothesis, although we cannot rule out the possibility that MG altered the kinetics of the actin-myosin interaction. Thus, despite strong evidence that tropomyosin and the troponin complex are not glycated by MG in vivo, their presence on the thin filament is required to observe an MG-dependent reduction in myosin binding to the thin filament. The observed decrease in myosin binding agrees with real-time synchrotron x-ray diffraction showing that in early diabetes, myosin heads are displaced from actin in beating heart muscle fibres (39). Given the need for a calcium-regulated thin filament to observe the functional impact of MG-modifications on actin and myosin, how might the reduction in calcium sensitivity at both the myocyte and myofilament level be related to these MG-modified proteins?

Calcium regulation and sensitivity of the myofilament system require shifts of tropomyosin on actin to expose myosin binding sites and subsequent myosin interaction with the thin filament to generate force and motion (40). In the absence of calcium, tropomyosin blocks these myosin binding sites, but upon calcium binding to the troponin complex, tropomyosin shifts position on actin, unmasking myosin binding sites. Once myosin binds strongly to the thin filament, tropomyosin shifts further on actin, resulting in the thin filament being fully activated. Therefore, calcium sensitivity of the thin filament is related to both actin- and myosin-dependent processes. As such, the reduced binding of MG-modified myosin to MG-modified NTFs in the cosedimentation assay could explain the reduction in calcium sensitivity observed in the in vitro motility assay when either NTFs or myosin was treated with MG. We will consider possible molecular mechanisms by which MG-modifications to either actin or myosin could result in the observed effect on calcium sensitivity.

On actin, we identified MG-modification of K293, a residue that is very near tropomyosin's position on F-actin in the absence of calcium, which blocks myosin binding. Two studies clearly illustrate how this region is critical for actin-tropomyosin interaction, as the authors mutated neighboring residues on actin 

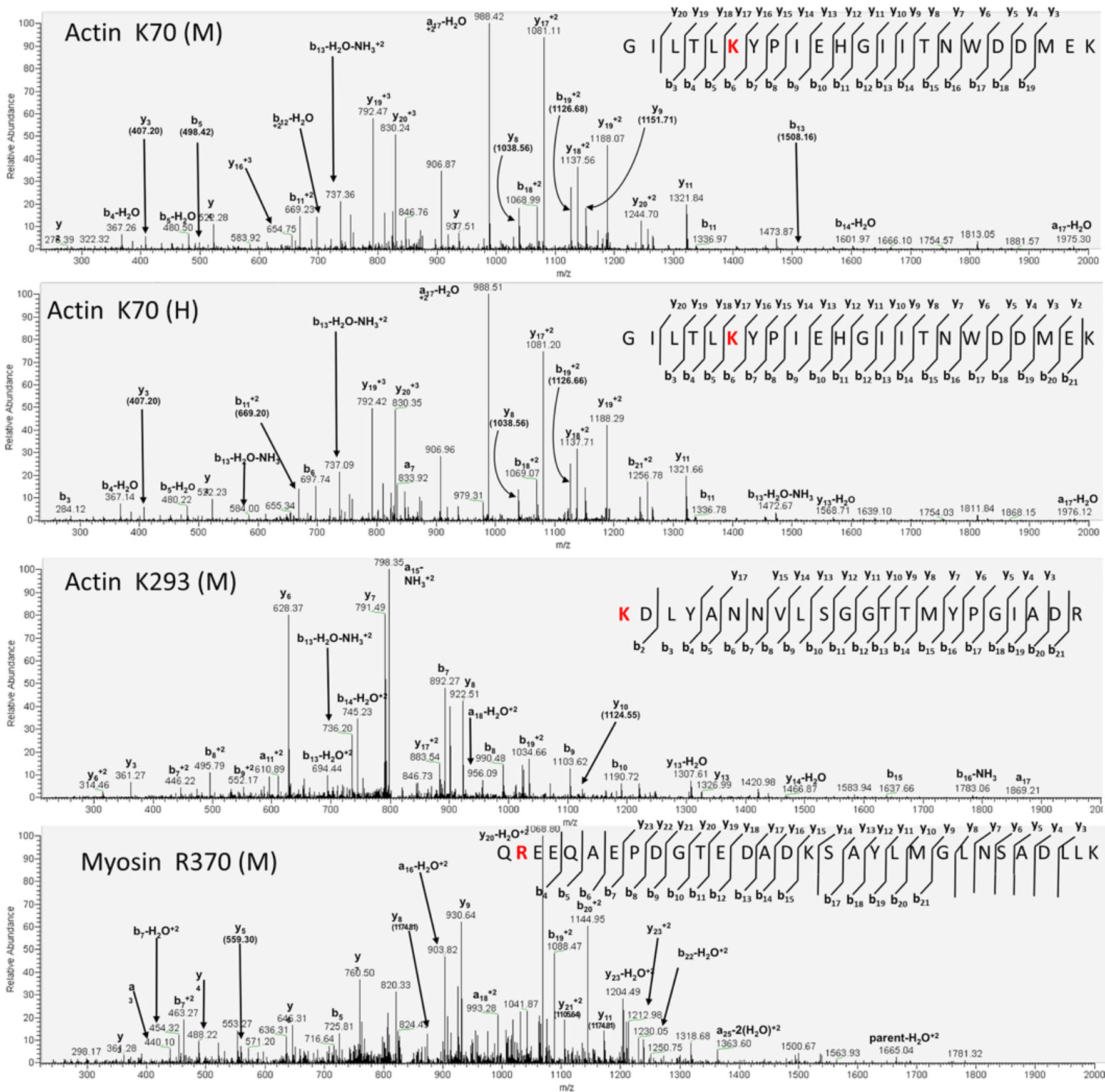

Figure 5. Spectra of peptides with methylglyoxal-modified sites identified by MS. Representative spectra of sites found to be modified by methylglyoxal (MC) on human $(H)$ and mouse $(M)$. The $b$ and $y$ ions are assigned to the spectra and the peptide sequence, with the $b$ and $y$ ions shown on the right of the spectra. The MG-modified site is shown in red. Actin K70 found in mouse is shown on the top, followed by the same site found in the human samples. In addition, actin K293 found in the mouse sample is shown and myosin R370 found on the mouse is also shown at the bottom. $n=3$ for mouse samples, $n=3$ for mouse samples treated with MG, $n=4$ for nonfailing human samples, and $n=5$ for diabetic heart failure human samples. MS was run 3 times for each sample.

(D294V and A297S by the numbering used here) and both disrupted tropomyosin's position on actin and affected calcium sensitivity $(41,42)$. Thus, it is highly likely that MG-modification of K293 would have a similar effect, explaining the observed decrease in calcium sensitivity.

For myosin, the MG-modification at R370 is in loop 4 of the myosin head, also called the cardiomyopathy loop. This site is in the region where myosin interacts with actin, partially by forming ionic bonds, to form a crossbridge $(43,44)$. Arginine and lysine residues, in addition to being MG targets, are positively charged and can form ionic bonds with negatively charged residues on the opposing protein in the 
A

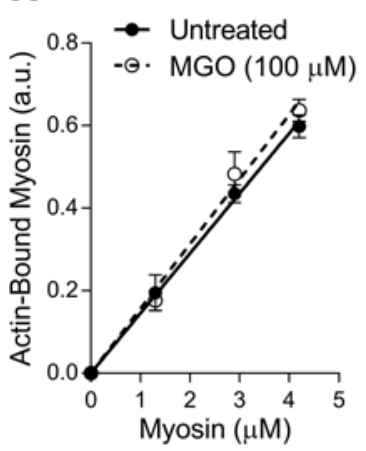

B

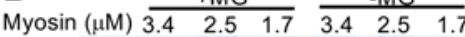

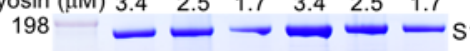
$\begin{array}{ll}{ }^{2.0} & \text { Cosedimentation between actin and } \\ & \text { myosin S1 fragment (S1), shown by }\end{array}$
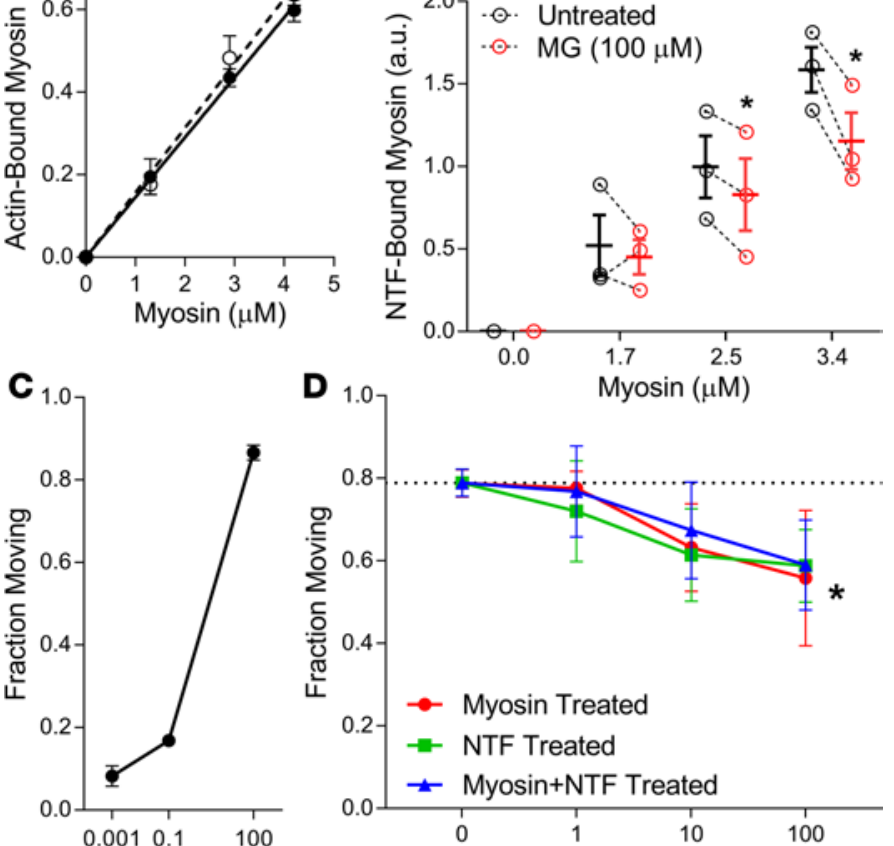

D

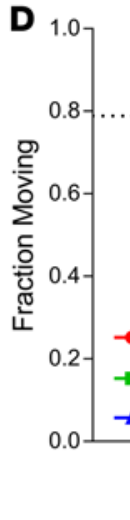

Calcium $(\mu \mathrm{M})$
Figure 6. Methylglyoxal reduces actin-myosin functional interaction mean + SEM and linear regression, was unaffected by treatment with $100 \mu \mathrm{M}$ methylglyoxal (MG) $(n=5)$.

(B) Representative cosedimentation of myosin $\mathrm{S} 1$ fragment (S1) and native thin filaments (NTFs) is shown (inset above, $n=3$ ) at $0.145 \mu \mathrm{M}$ calcium and individual data points before (black) and after (red) $100 \mu \mathrm{M} \mathrm{MG}$ treatment (dashed lines connect the same sample before and after treatment) and mean \pm SEM. ${ }^{*} P<$ 0.05 by 2-way repeated-measures ANOVA and Holm-Sidak post hoc test. (C) Fraction moving for NTFs at different calcium concentrations in the in vitro motility assay $(n=4-5$ at each concentration) (mean \pm SEM). (D) NTF fraction moving at $0.175 \mu \mathrm{M}$ calcium where either myosin (red), NTFs (green), or both (blue) were treated with $1 \mu \mathrm{M}, 10, \mu \mathrm{M}$, or $100 \mu \mathrm{M}$ MG ( $n=5$ per group) (mean \pm SEM). ${ }^{*} P<0.05$ for MG treatment (for all 3 groups) by 2-way repeated-measures ANOVA and Holm-Sidak post hoc test. (E) Individual data points as well as corresponding mean \pm SEM for NTF fraction moving as in $\mathbf{D}$.

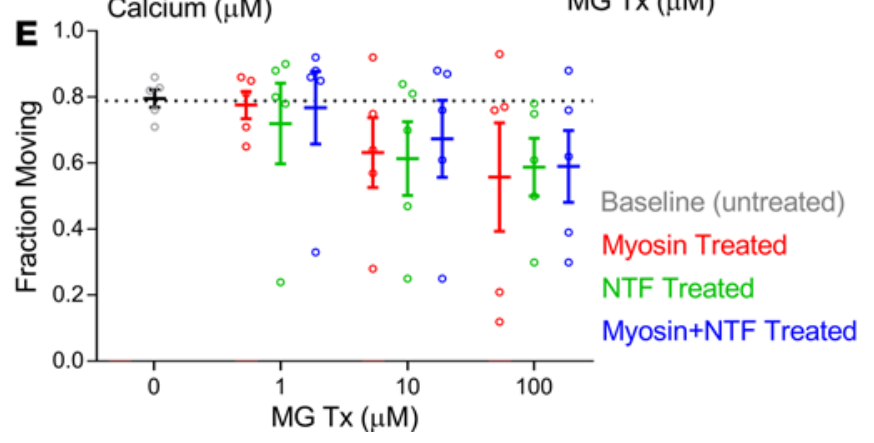

actin-myosin contact zone. Previous analysis of the actin-myosin interface suggests R370 on myosin creates a H-bond with K328 on actin, and an electrostatic interaction with D311 on actin (43). The MG-modification at R370 may prevent myosin from binding as strongly to actin, which is normally required for fully activating the thin filament. Furthermore, there is evidence that in rigor, myosin can directly interact with tropomyosin (45), and tropomyosin's position on the thin filament can still be regulated by calcium (46). Thus, when myosin is bound to actin it may interact with tropomyosin in a calcium-dependent manner, which may be required to fully activate the thin filament, offering another possible explanation for how myosin MG-modifications might affect calcium sensitivity.

We also discovered 2 MG-modifications on myosin that were increased in dbHF compared with NF at residues with known disease-causing mutations, K384 and K1899. Myosin K384 is a highly conserved residue, and in the predominant human isoform, $\beta$-MHC, a missense mutation at this residue (K383N) results in hypertrophic cardiomyopathy (47). This mutation to an asparagine would result in an uncharged side chain, as MG-modification would. Likewise, K1899C in $\alpha-\mathrm{MHC}$, in the coiled-coil region of the protein, results in familial dilated cardiomyopathy (48). That two of the MG-modifications we discovered are at sites where mutations result in cardiomyopathy further underscores the importance of these residues to muscle function and their likelihood of inducing disease when modified.

The MG-modified sites on myosin were assigned to isoforms that are not highly expressed in human cardiac muscle, such as $\alpha-\mathrm{MHC}$ and embryonic skeletal MHC. Even at low levels, however, $\alpha-\mathrm{MHC}$ can have dramatic effects on function (49), maintaining the possible functional relevance of these modifications. The 
peptides identified by MS had very high homology with $\beta$-MHC (Supplemental Figure 4) but were nonetheless unambiguously from different MHC isoforms. Furthermore, MS analysis detected peptides from $\beta$-MHC that include these residues (R369, R383, and K1898), and all were unmodified. Given the high homology between $\alpha$ - and $\beta$-MHC (93\%), especially in these regions, it is surprising that MG would modify one and not the other. Further studies are needed to understand this selectivity.

The physiological and pathological concentrations of MG are still controversial. Physiological MG plasma concentrations are reported between $100 \mathrm{nM}$ and $2 \mu \mathrm{M}(50,51)$ and tissue concentrations are between $2-4$ $\mu \mathrm{M}$ (28). Diabetes appears to elevate these levels by 2- to 4-fold (52), although greater changes have also been reported. We detected no functional effect of $1 \mu \mathrm{MMG}$, supporting this concentration at or near physiological levels. While it is difficult to estimate chronic in vivo concentrations from acute in vitro experiments, our data support a human pathological cardiac tissue concentration somewhere between 10 and $100 \mu \mathrm{M}$, since MG had no further effect on calcium sensitivity between those two doses in dbHF. Since MG-modifications are irreversible and not in equilibrium, many in vitro studies use much higher concentrations (100 $\mu \mathrm{M}$ to $1 \mathrm{mM})$ to represent the effects of chronic exposure to $\mathrm{MG}(53,54)$. The cumulative effect of irreversible MG-modifications is especially important for myofilament proteins, which have slower turnover rates than most protein structures in the cell (55) and will thus accrue more modifications before their degradation. When we treated mouse cardiomyocytes with $100 \mu \mathrm{M}$ MG acutely we were unable to detect a significant increase in MG-modifications using MS, although we were able to detect increased modifications in the chronic human dbHF. This difference supports the concept that much higher doses of acute exposure to MG are necessary to represent the effects of chronic pathological exposure in vivo. Indeed, while the $100 \mathrm{mM}$ MG treatment used for the mouse MS experiment is 1,000-fold higher than most estimates of in vivo pathological concentrations, we only detected $68 \%$ more MG-modifications compared with the human dbHF samples. These results indicate that our in vitro treatment concentrations result in similar levels of the actual modifications that MG induces, irrespective of the absolute blood and tissue concentrations, which still lack consensus. We are therefore confident that the concentrations used are physiologically and pathologically relevant functionally and structurally.

Previous studies show that diabetes is associated with decreased myofilament calcium sensitivity, although there are several studies that show no change, or even an increase $(56,57)$. When multiple concomitant cardiovascular diseases overlap, the end result can be a superposition of the individual effects, and heart failure alone has been shown to induce an increase in calcium sensitivity $(58,59)$. Thus, the result on myofilament function may depend greatly on the specific patient population or model used. Here we focused on severe, chronic $\mathrm{dbHF}$ (these were patients receiving heart transplants) and acute exposure to pathologically relevant doses of MG. In our human functional experiments, dbHF cardiomyocytes were insensitive to functional changes from high doses of MG, as they already have increased baseline modifications compared with the NF samples. As we identified MG-modifications in both models, and a significant acute functional impact, we find it likely this mechanism has an important impact on the transition to $\mathrm{dbHF}$, even if it is masked by severe comorbidities eventually. Our results suggest that MG-modification of the myofilament could contribute to the earliest development of heart failure in diabetic patients. In fact, previous studies have shown that chronic administration of MG alone results in a diabetic cardiomyopathy-like phenotype, supporting its central pathogenic role (60).

The patient samples that were studied here represent a diverse group that have 2 things in common: diabetes and heart failure. Two of the 6 patients had diabetic cardiomyopathy, which is defined as diabetes with ventricular dysfunction in the absence of coronary atherosclerosis and hypertension (14). Four had ischemia, and 5 of the 6 had diastolic dysfunction. Thus, the accumulation of MG-modifications on the sarcomere, and the negative functional consequence, appears to be independent of a range of other complications. However, those patients with diabetic cardiomyopathy may see the largest beneficial impact of targeting these modifications or their functional effects, since they have fewer additional complications also contributing to the development of heart failure. Importantly, patients with DCM showed no increase in total myofilament MG-modifications, suggesting this mechanism is specific to diabetes and not to heart failure in general.

There has been substantial interest in AGE-breakers, drugs that were thought to be able to reduce AGE modifications, as a treatment for cardiovascular disease in diabetic patients $(61,62)$. Unfortunately, the results of the clinical trials have not supported the efficacy of this approach (25). As patients with $\mathrm{dbHF}$ are a heterogeneous population with a myriad of comorbidities, it would be challenging to treat them with such broadly targeted approaches. The results from this study suggest a way forward, as the myofilament is a pharmacologically tuneable system with calcium sensitizers (63), myosin activators like 
omecamtiv mecarbil (26), and others (64). We have found that diabetes depresses myofilament function via MG-modifications and we therefore have a toolbox to correct the detrimental effects clinically. Furthermore, if these modifications are pathogenic, these compounds might help avoid the development, or ameliorate the consequences, of heart failure in the growing diabetic patient population.

\section{Methods}

Human samples. Deidentified human LV heart tissue samples from 7 control and $6 \mathrm{dbHF}$ patients were used for this study. Control samples were obtained from organ donors who did not have diabetes when the heart could not be transplanted into another patient. dbHF samples were obtained from patients who had diabetes and who received heart transplants.

Dot blot. Myofilament fractions were prepared from human heart left ventricle by homogenizing approximately $200 \mu \mathrm{g}$ of tissue in a glass homogenizer in a standard rigor buffer (SRB) (58), $1 \%$ Triton, and protease and phosphatase inhibitors. After washing out the Triton, the pellet was then resuspended in $8 \mathrm{M}$ urea with $1 \%$ SDS. Protein $(50 \mu \mathrm{g})$ was used for the dot blot. Due to the large volume of sample required, 3 individual blots were used for the experiment. The antibodies used were anti-methylglyoxal (Cell Biolabs, STA-011) and anti- $\alpha$-tubulin (Cell Signaling Technology, 11H10). The membranes were scanned using an Odyssey infrared imager (Licor) at 700 and $800 \mathrm{~nm}$. Images were analyzed using the Licor software and were within the linear range.

$M S$. LV samples from human control and dbHF patients were homogenized in relaxing solution containing (in mM): $5.95 \mathrm{Na}_{2} \mathrm{ATP}, 6.41 \mathrm{MgCl}_{2}, 10$ EGTA, $100 \mathrm{BES}, 10$ phosphocreatine, 50.25 KProp, 10 DTT, with $0.3 \%$ Triton and protease and phosphatase inhibitors (Thermo Fisher Scientific). The myofilament pellets were resuspended in $8 \mathrm{M}$ urea, 1\% SDS and underwent trypsin digestion using the FASP protocol (59). Alternatively, C57BL/6J mouse LV samples were homogenized in relaxing solution (with protease and phosphatase inhibitors) and centrifuged, and the pellet was resuspended with relaxing solution or MG at a specific concentration for 20 minutes after washing out the Triton. The myofilament fraction was prepared as described above and $100 \mu \mathrm{g}$ of tissue lysate was digested with $2.4 \mu \mathrm{g}$ of trypsin and incubated at $37^{\circ} \mathrm{C}$ for 3 hours. Human samples were run twice under 2 different digestion protocols. For the first run, $400 \mu \mathrm{g}$ of tissue lysate was digested with $4 \mu \mathrm{g}$ of trypsin at $37^{\circ} \mathrm{C}$ overnight and for the second run $200 \mu \mathrm{g}$ of tissue lysate was digested using $12.5 \mu \mathrm{g}$ of trypsin for 3 hours at $37^{\circ} \mathrm{C}$. LC-MS/MS analysis was performed using an Ultimate 3000 Nano LC (Thermo Fisher Scientific) connected to an Orbitrap Elite mass spectrometer (Thermo Fisher Scientific) equipped with an EasySpray ion source. Peptides were first loaded onto a trap column (PepMap100 C18, $5 \mu \mathrm{m}, 100 \AA, 300 \mu \mathrm{m}$ i.d. $\times 5 \mathrm{~mm}$, Thermo Fisher Scientific) followed by separation in a PepMap RSLC C18 column $(2 \mu \mathrm{m}, 100 \AA$, $75 \mu \mathrm{m}$ i.d. $\times 25 \mathrm{~cm}$, Thermo Fisher Scientific) using a flow rate of $300 \mathrm{nl} / \mathrm{min}$ with a linear gradient of $5 \%-35 \%$ B for 90 minutes, 35\%-95\% $\mathrm{B}$ for 3 minutes, holding at 95\% B for 7 minutes, and re-equilibrating at 5\% B for 25 minutes at $400 \mathrm{nl} / \mathrm{min}$ (mobile phase A was $0.1 \%$ formic acid in water and mobile phase B was $0.1 \%$ formic acid in acetonitrile).

For the myofilament samples (100 $\mu$ g protein, $12 \mu \mathrm{g}$ trypsin), the flow rate was $5 \%-35 \%$ B for 60 minutes, $35 \%-95 \%$ B for 3 minutes, holding at $95 \%$ B for 7 minutes, and re-equilibrating at 5\% B for 25 minutes at $400 \mathrm{nl} / \mathrm{min}$. The nano-source capillary temperature was set to $275^{\circ} \mathrm{C}$ and the spray voltage was set to 2 $\mathrm{kV}$. MS1 scans were acquired in the Orbitrap Elite at a resolution of 60,000 full width at half maximum (FWHM) (400-1,700 m/z) with an automatic gain control (AGC) target of $1 \times 10^{6}$ ions over a maximum of $250 \mathrm{~ms}$. The top 15 ions from the MS1 scan were selected for fragmentation in the ion trap using CID with a normalized collision energy of $35 \%$ and analyzed using rapid scan rates in the ion trap with a target setting of $1 \times 10^{4}$ ions, an accumulation time of $100 \mathrm{~ms}$, and an isolation width of $2 \mathrm{Da}$. Monoisotopic precursor selection was enabled and only MS1 signals exceeding 500 counts triggered the MS2 scans, with +1 and unassigned charge states not being selected for MS2 analysis. Dynamic exclusion was enabled with a repeat count of 2 , repeat duration of 30 seconds, and exclusion duration of 90 seconds.

In-gel digestion and MS analysis was also performed on specific smaller proteins. $\mathrm{NF}(n=4)$ and $\mathrm{dbHF}$ $(n=4)$ myofilament-enriched samples were run in a $4 \%-12 \%$ SDS-PAGE gel, and then individual bands were cut out from each sample for the proteins of interest (tropomyosin, troponin I, troponin T, and troponin T). Each band from each sample (32 total) was individually digested, desalted, and analyzed by MS. LC-MS/MS analysis was performed using an Ultimate 3000 Nano LC connected to an Orbitrap LUMOS mass spectrometer equipped with an EasySpray ion source. Peptides were loaded onto a PepMap RSLC C18 column $(2 \mu \mathrm{m}, 100 \AA, 75 \mu \mathrm{m}$ i.d. $\times 25 \mathrm{~cm})$ using a flow rate of $300 \mathrm{nl} / \mathrm{min}$ for 15 minutes at $1 \% \mathrm{~B}$ 
(mobile phase A was $0.1 \%$ formic acid in water and mobile phase B was $0.1 \%$ formic acid in acetonitrile), after which point they were separated with a linear gradient of $5 \%-35 \%$ B for 11 minutes, $35 \%-85 \%$ B for 3 minutes, holding at $85 \% \mathrm{~B}$ for 5 minutes, and re-equilibrating at $1 \% \mathrm{~B}$ for 17 minutes at $400 \mathrm{nl} / \mathrm{min}$ The nano-source capillary temperature was set to $300^{\circ} \mathrm{C}$ and the spray voltage was set to $1.8 \mathrm{kV}$. MS1 scans were acquired in the Orbitrap at a resolution of $120,000 \mathrm{~Hz}$ from mass range 400-1,600 $\mathrm{m} / \mathrm{z}$. For MS1 scans the AGC target was set to $4 \times 10^{5}$ ions with a max fill time of $50 \mathrm{~ms}$. MS2 spectra were acquired using the TopSpeed method with a total cycle time of 3 seconds and an AGC target of $1 \times 10^{4}$ and a max fill time of $100 \mathrm{~ms}$, and an isolation width of $1.6 \mathrm{Da}$ in the quadrapole. Precursor ions were fragmented using HCD with a normalized collision energy of $30 \%$ and analyzed using rapid scan rates in the ion trap. Monoisotopic precursor selection was enabled and only MS1 signals exceeding 5,000 counts triggered the MS2 scans, with +1 and unassigned charge states not being selected for MS2 analysis. Dynamic exclusion was enabled with a repeat count of 1 and exclusion duration of 60 seconds.

MS data analysis. All raw data were searched using the Sorcerer-Sequest algorithm (Sagen) with postprocessing using Scaffold v4.4.6 and Scaffold PTM v2.1.3 (Proteome Software) using the most recent version of either the human or mouse UniProt database. The following search parameters were used: full trypsin cleavage, static modification of $+57 \mathrm{Da}$ on cysteine, variable modification of $+16 \mathrm{Da}$ on methionine +16 on $\mathrm{M}$ (oxidation), +54 Da on R (MG-H1) and +72 Da on K (carboxyethyl), MS1 error of 10 ppm, MS2 error of $1 \mathrm{Da}$. Peptide confidence was set to $95 \%$ and localization probability of $90 \%$ was required for inclusion of a modification site in the quantitative data set.

Skinned myocytes. Myocytes were prepared by homogenizing C57BL/6J (Jackson Labs) mouse left ventricles with $0.3 \%$ Triton and protease and phosphatase inhibitors (Sigma-Aldrich) in isolation solution and left to skin on ice. Isolation solution consisted of (in mM): $5.5 \mathrm{Na}_{2} \mathrm{ATP}, 7.11 \mathrm{MgCl}_{2}, 2 \mathrm{EGTA}, 108.01 \mathrm{KCl}$, $8.91 \mathrm{KOH}, 10$ imidazole, $10 \mathrm{DTT}$. After skinning, the mixture was centrifuged and the pellet was resuspended twice in isolation solution to remove the detergent. A single myocyte was selected and attached to a force transducer and a length controller (Aurora Scientific). The sarcomere length was monitored using a video camera and calculated by the High-Speed Video Sarcomere Length software (Aurora Scientific) and was kept constant at $2.1 \mu \mathrm{m}$ throughout the experiment. The temperature was kept constant at $25^{\circ} \mathrm{C}$. For the passive tension experiments, the sarcomere length was varied from 1.8 to $2.4 \mu \mathrm{m}$.

The myocyte was kept in relaxing solution with phosphatase and protease inhibitors. The force was measured by moving the myocytes to different baths containing different calcium concentrations ranging from 0 to saturating conditions, created by mixing relaxing and activation solution. Activation solution contained (in mM): $5.95 \mathrm{Na}_{2} \mathrm{ATP}, 6.2 \mathrm{MgCl}_{2}, 10 \mathrm{Ca}^{2+}$-EGTA, $100 \mathrm{BES}, 10$ phosphocreatine, $29.98 \mathrm{KProp}$, 10 DTT, phosphatase and protease inhibitors. The same myocyte was then incubated with MG solution in relaxing solution for 20 minutes in a separate bath and another force-calcium curve was produced. For the control myocytes, each one was incubated in relaxing solution for 20 minutes.

The Hill equation,

$F=F_{\max }\left(\frac{\left[\mathrm{Ca}^{2+}\right]^{h}}{\mathrm{EC}_{30}{ }^{h+}\left[\mathrm{Ca}^{2+}\right]^{h}}\right)$

was fitted to the force-calcium data to acquire the parameters for $\mathrm{F}_{\max }$, calcium sensitivity $\left(\mathrm{EC}_{50}\right)$, and Hill coefficient $(h)$. This experiment yields 3 parameters: $\mathrm{F}_{\max }$, which is the maximal force of the myocyte; $\mathrm{EC}_{50}$, which is an indicator of the calcium sensitivity; and $h$, the cooperativity of activation. For all the myocyte experiments, individual myocytes were used from at least 3 biological samples.

Cosedimentation assay. Rabbit skeletal muscle actin (Cytoskeleton, Inc., AKL-99A) was reconstituted to $1 \mathrm{mg} / \mathrm{ml}$ in cold general actin buffer $\left(5 \mathrm{mM}\right.$ Tris- $\left.\mathrm{HCl} \mathrm{pH} 8.0,0.2 \mathrm{mM} \mathrm{CaCl}_{2}\right)$ and incubated on ice for 30 minutes. Actin polymerization buffer $(500 \mathrm{mM} \mathrm{KCl}, 20 \mathrm{mM} \mathrm{MgCl}, 10 \mathrm{mM}$ ATP) was added in a 1:10 (v/v) ratio, followed by incubation at room temperature for 1 hour. Bovine cardiac thin filament complex (Cytoskeleton, Inc., CS-TFC01) was reconstituted to $1 \mathrm{mg} / \mathrm{ml}$ in PIPES buffer (16.8 mM PIPES pH 7.4, $2.8 \mathrm{mM} \mathrm{MgCl}_{2}, 2 \%[\mathrm{w} / \mathrm{v}]$ sucrose) and incubated at room temperature for 10 minutes. Myosin motor protein S1 fragment (Cytoskeleton, Inc., CS-MYS04-A) was reconstituted to $1.9 \mathrm{mg} / \mathrm{ml}$ in water, generating a $21 \mu \mathrm{M}$ stock of S1 myosin in the following buffer: $20 \mathrm{mM}$ PIPES pH 7.0, $30 \mathrm{~mm} \mathrm{KCl}, 1 \mathrm{mM}$ EDTA, 5\% $(\mathrm{w} / \mathrm{v})$ sucrose, and $1 \%(\mathrm{w} / \mathrm{v})$ dextran.

For actin-myosin cosedimentation, actin was added to 6 tubes in a constant volume $(10 \mu 1)$ and to 3 of these $100 \mu \mathrm{M}$ MG was added, followed by incubation for 5 minutes at room temperature. Myosin in varying concentrations $(4.2 \mu \mathrm{M}, 3.0 \mu \mathrm{M}, 1.3 \mu \mathrm{M})$ was added to the MG-treated and -untreated tubes. 
Myosin alone in buffer and actin alone served as controls. All tubes were incubated at room temperature for 30 minutes. All tubes were spun at $150,000 \mathrm{~g}$ for 1.5 hours at $24^{\circ} \mathrm{C}$ in an Optima TLX Ultracentrifuge (Beckman Coulter).

For NTF-myosin cosedimentation, NTFs (Cytoskeleton, Inc.) were added in a constant volume (10 $\mu$ l) to 13 tubes. Tube 1 served as control and consisted of thin filaments in PIPES buffer. Tubes 2 and 3 were assigned low calcium and received high myosin $(3.4 \mu \mathrm{M})$ and low myosin $(1.7 \mu \mathrm{M})$, respectively. Tubes 4-6 were assigned medium calcium and received high, medium $(2.5 \mu \mathrm{M})$, and low myosin with $\mathrm{MG}(100 \mu \mathrm{M})$. Tubes 7-9 matched these but were not treated with MG. Tubes 10 and 11 were assigned high calcium and received high and low myosin with MG. Tubes 12 and 13 matched these but were not treated with MG. All tubes were incubated at room temperature for 1 hour. All tubes were spun at $150,000 \mathrm{~g}$ for 1.5 hours at $24^{\circ} \mathrm{C}$ in an Optima TLX Ultracentrifuge.

Following centrifugation, the supernatants and pellets were separated and the pellets resuspended in 10 $\mu 1$ of Milli-Q water and incubated on ice for 10 minutes. Tris-glycine SDS loading dye and Bolt reducing buffer (Thermo Fisher Scientific) were added to each sample and the supernatant and pellet samples were run in an SDS-PAGE gel. The gel was stained with Coomassie blue. Resulting protein bands were quantified by calculating the ratio of myosin to actin in each lane using ImageJ software (NIH).

In vitro motility assay. The in vitro motility assay, in which mouse cardiac NTFs were observed moving over a surface of mouse cardiac myosin adhered to a glass coverslip within a $20-\mu 1$, nitrocellulose-coated flow cell at $22^{\circ} \mathrm{C}$, has been described previously (65). Briefly, ventricular cardiac myosin $(100 \mu \mathrm{g} / \mathrm{ml})$ in myosin buffer (300 mM KCl, 1 mM EGTA, $10 \mathrm{mM} \mathrm{DTT,} 25 \mathrm{mM}$ imidazole, $4 \mathrm{mM} \mathrm{MgCl}$, pH 7.4) was incubated in the flow cell ( 2 minutes), followed by BSA $(1 \mathrm{mg} / \mathrm{ml})$ in actin buffer (AB: $25 \mathrm{mM} \mathrm{KCl}, 1 \mathrm{mM} \mathrm{EGTA}, 10 \mathrm{mM}$ DTT, $25 \mathrm{mM}$ imidazole, $4 \mathrm{mM} \mathrm{MgCl}{ }_{2}, \mathrm{pH}$ 7.4) to block the nitrocellulose surface. Unlabeled NTFs $(1 \mu \mathrm{M}$ in $\mathrm{AB})$ were then infused into the flow cell and incubated for 1 minute in order to eliminate denatured myosin heads on the surface that irreversibly bind actin. Following this incubation, $40 \mu \mathrm{l}$ of AB with $1 \mathrm{mM}$ ATP was infused into the flow cell to release any of the NTFs not bound to the denatured myosin, followed by $40 \mu \mathrm{l}$ of AB. Then, $40 \mu \mathrm{l}$ of $1 \mu \mathrm{M}$ tetramethyl-rhodamine-phalloidin-labeled NTFs in AB was added to the flow cell for 1 minute and rinsed 3 times with AB to wash out any unbound NTFs. To observe NTF movement in a calcium-dependent manner, the final motility buffer (MB: AB with $100 \mu \mathrm{M}$ ATP, $0.5 \%$ methylcellulose, and various $\left[\mathrm{CaCl}_{2}\right]$ ) was infused into the flow cell. The $\mathrm{MB}$ calcium concentration was determined using MaxChelator software (66) so that the free $\mathrm{Ca}^{2+}$, described as $\mathrm{pCa}\left(-\log \left[\mathrm{Ca}^{2+}\right]\right)$, ranged from pCa 4-9 (65).

The effect of MG on $\mathrm{Ca}^{2+}$-dependent NTF motility was characterized by treating NTFs and myosin with varying $M G$ concentrations prior to the in vitro motility assay. Specifically, fluorescently labeled NTFs $(1 \mu \mathrm{M}$ in $\mathrm{AB})$ and myosin $(0.2 \mu \mathrm{M}$ in myosin buffer) were incubated with 1,10 , or $100 \mu \mathrm{M}$ MG for 20 minutes at room temperature prior to use. Fluorescence microscopy was performed using a Nikon Eclipse TiU microscope equipped with a Plan Apo objective $(\times 100,1.35$ numerical aperture), Lumen $200 \mathrm{~W}$ metal arc lamp (Prior Scientific), and a Mega Z 10-bit digital camera (Stanford Photonics). Images were acquired using Piper software at 10 frames/second without pixel binning (93 nm/pixel) and downsampled to 2 frames/second using ImageJ $1.43 \mathrm{u}(\mathrm{NIH})$. Images were analyzed for the percentage of motile NTFs in each movie using DiaTrack 3.03 software (Semasopht).

ProQ and SYPRO Ruby staining. Twenty micrograms of myofilament fraction of control and treated samples (as described above) was run in an SDS-PAGE gel at $180 \mathrm{~V}$ for 45 minutes. The gel was placed directly onto $100 \mathrm{ml}$ of fixing solution (50\% methanol, 10\% acetic acid) for 60 minutes and then stained for 60 minutes with ProQ Diamond (Invitrogen) stain. The gel was destained 3 times for 30 minutes with destain solution (50 mM sodium acetate $\mathrm{pH}$ 4.0/acetonitrile) and imaged on a Typhoon 9410 imager (GE) with a CY3 filter set. To visualize total protein, the gel was placed on SYPRO Ruby stain (Invitrogen) overnight. The next day, the gel was destained with destain solution (18\% ethanol/ $7 \%$ acetic acid) and imaged using the Typhoon imager.

Statistics. Unless otherwise indicated, data were compared within the groups using 2-way ANOVA with post hoc comparisons made with the Holm-Sidak method using GraphPad Prism version 6.0. MS/MS spectral counts were compared using paired (for mouse data) or unpaired (for human data) Student's $t$ test. Patient characteristics statistics were evaluated by $t$ test or Fisher's exact test. All tests were 2-tailed and a $P$ value of less than 0.05 was considered significant. All values are represented as the mean \pm SEM.

Study approval. Human study protocols were approved by the IRBs at the University of Kentucky and the Cleveland Clinic. All patients gave informed consent. Animal studies were approved by the Loyola University Chicago Health Sciences Division Institutional Animal Care and Use Committee. 


\section{Author contributions}

MP, RJH, and JAK conceptualized the project. MP, RJH, SBP, DMW, and JAK designed the research studies. MP, RJH, SBP, AL, TGM, and MJS performed experiments and acquired data. MP, RJH, SBP, TGM, and JAK analyzed data and created the figures. KSC, CAB, and CSM provided human samples. MP and JAK wrote the manuscript. RJH, JEVE, KSC, and DMW revised the manuscript.

\section{Acknowledgments}

We would like to thank Nikolai Smolin and Seth Robia from Loyola University Chicago and Anthony Cammarato from Johns Hopkins University for helpful discussions on protein structure. This work was supported by the NIH (R01HL136737 to JAK and R01HL126909 to DMW) and the American Heart Association (14SDG20380148 to JAK and 111POST7210031 to MP). We would like to thank the patients and organ donors who donated the samples in this project and the Cardiovascular Biorepository at the University of Kentucky.

Address correspondence to: Jonathan A. Kirk, Department of Cell and Molecular Physiology, Loyola University Chicago Stritch School of Medicine, Center for Translational Research, Room 522, 2160 S. First Avenue, Maywood, Illinois 60153, USA. Phone: 708.216.6348; Email: jkirk2@1uc.edu.

1. National Diabetes Statistics Report, 2017. Centers for Disease Control and Prevention. https://www.cdc.gov/diabetes/pdfs/ data/statistics/national-diabetes-statistics-report.pdf. Accessed September 17, 2018.

2. Gu K, Cowie CC, Harris MI. Mortality in adults with and without diabetes in a national cohort of the U.S. population, 1971-1993. Diabetes Care. 1998;21(7):1138-1145.

3. Kannel WB, Hjortland M, Castelli WP. Role of diabetes in congestive heart failure: the Framingham study. Am J Cardiol. 1974;34(1):29-34

4. Shah MS, Brownlee M. Molecular and cellular mechanisms of cardiovascular disorders in diabetes. Circ Res. 2016;118(11):1808-1829.

5. Willemsen S, et al. The role of advanced glycation end-products and their receptor on outcome in heart failure patients with preserved and reduced ejection fraction. Am Heart J. 2012;164(5):742-749.e3.

6. Hovatta I, et al. Glyoxalase 1 and glutathione reductase 1 regulate anxiety in mice. Nature. 2005;438(7068):662-666

7. Jakubcakova V, Curzi ML, Flachskamm C, Hambsch B, Landgraf R, Kimura M. The glycolytic metabolite methylglyoxal induces changes in vigilance by generating low-amplitude non-REM sleep. J Psychopharmacol (Oxford). 2013;27(11):1070-1075

8. Rabbani N, Thornalley PJ. Dicarbonyl proteome and genome damage in metabolic and vascular disease. Biochem Soc Trans. 2014;42(2):425-432

9. Thornalley PJ. The glyoxalase system in health and disease. Mol Aspects Med. 1993;14(4):287-371.

10. Thornalley PJ, et al. The human red blood cell glyoxalase system in diabetes mellitus. Diabetes Res Clin Pract. 1989;7(2):115-120.

11. Yuen A, et al. Methylglyoxal-modified collagen promotes myofibroblast differentiation. Matrix Biol. 2010;29(6):537-548

12. Stratmann B, Goldstein B, Thornalley PJ, Rabbani N, Tschoepe D. Intracellular accumulation of methylglyoxal by glyoxalase 1 knock down alters collagen homoeostasis in L6 myoblasts. Int J Mol Sci. 2017;18(3):E480.

13. Blackburn NJR, et al. Methylglyoxal-derived advanced glycation end products contribute to negative cardiac remodeling and dysfunction post-myocardial infarction. Basic Res Cardiol. 2017;112(5):57.

14. Jia G, Hill MA, Sowers JR. Diabetic cardiomyopathy: An update of mechanisms contributing to this clinical entity. Circ Res. 2018;122(4):624-638.

15. Nam DH, et al. CHOP deficiency prevents methylglyoxal-induced myocyte apoptosis and cardiac dysfunction. J Mol Cell Cardiol. $2015 ; 85: 168-177$.

16. Shao $\mathrm{CH}$, et al. Carbonylation contributes to SERCA2a activity loss and diastolic dysfunction in a rat model of type 1 diabetes. Diabetes. 2011;60(3):947-959.

17. Shao $\mathrm{CH}$, et al. Carbonylation induces heterogeneity in cardiac ryanodine receptor function in diabetes mellitus. Mol Pharmacol. 2012;82(3):383-399.

18. Tian C, et al. Reactive carbonyl species and their roles in sarcoplasmic reticulum $\mathrm{Ca}^{2+}$ cycling defect in the diabetic heart. Heart Fail Rev. 2014;19(1):101-112.

19. Zhang $\mathrm{L}$, et al. Inhibition of protein kinase $\mathrm{C} \beta \mathrm{II}$ isoform ameliorates methylglyoxal advanced glycation endproduct-induced cardiomyocyte contractile dysfunction. Life Sci. 2014;94(1):83-91.

20. Shao CH, et al. Carbonylation of myosin heavy chains in rat heart during diabetes. Biochem Pharmacol. 2010;80(2):205-217.

21. Jweied EE, et al. Depressed cardiac myofilament function in human diabetes mellitus. Am J Physiol Heart Circ Physiol. 2005;289(6):H2478-H2483.

22. Rundell VL, Geenen DL, Buttrick PM, de Tombe PP. Depressed cardiac tension cost in experimental diabetes is due to altered myosin heavy chain isoform expression. Am J Physiol Heart Circ Physiol. 2004;287(1):H408-H413.

23. Wickley PJ, Shiga T, Murray PA, Damron DS. Propofol decreases myofilament Ca2+ sensitivity via a protein kinase C-, nitric oxide synthase-dependent pathway in diabetic cardiomyocytes. Anesthesiology. 2006;104(5):978-987.

24. LeWinter MM. Functional consequences of sarcomeric protein abnormalities in failing myocardium. Heart Fail Rev. 2005;10(3):249-257

25. Engelen L, Stehouwer CD, Schalkwijk CG. Current therapeutic interventions in the glycation pathway: evidence from clinical 
studies. Diabetes Obes Metab. 2013;15(8):677-689.

26. Malik FI, et al. Cardiac myosin activation: a potential therapeutic approach for systolic heart failure. Science. 2011;331(6023):1439-1443.

27. Thornalley PJ, et al. Quantitative screening of advanced glycation endproducts in cellular and extracellular proteins by tandem mass spectrometry. Biochem J. 2003;375(Pt 3):581-592.

28. Rabbani N, Thornalley PJ. Methylglyoxal, glyoxalase 1 and the dicarbonyl proteome. Amino Acids. 2012;42(4):1133-1142.

29. Miki T, Yuda S, Kouzu H, Miura T. Diabetic cardiomyopathy: pathophysiology and clinical features. Heart Fail Rev. 2013;18(2):149-166.

30. Zile MR, et al. Myocardial stiffness in patients with heart failure and a preserved ejection fraction: contributions of collagen and titin. Circulation. 2015;131(14):1247-1259.

31. Hopf AE, et al. Diabetes-induced cardiomyocyte passive stiffening is caused by impaired insulin-dependent titin modification and can be modulated by neuregulin-1. Circ Res. 2018;123(3):342-355.

32. Ahmed N, Argirov OK, Minhas HS, Cordeiro CA, Thornalley PJ. Assay of advanced glycation endproducts (AGEs): surveying AGEs by chromatographic assay with derivatization by 6-aminoquinolyl-N-hydroxysuccinimidyl-carbamate and application to Nepsilon-carboxymethyl-lysine- and Nepsilon-(1-carboxyethyl)lysine-modified albumin. Biochem J. 2002;364(Pt 1):1-14

33. Previs MJ, et al. Myosin-binding protein C corrects an intrinsic inhomogeneity in cardiac excitation-contraction coupling. Sci Adv. 2015;1(1):e1400205.

34. Turk Z. Glycotoxines, carbonyl stress and relevance to diabetes and its complications. Physiol Res. 2010;59(2):147-156

35. Kirk JA, et al. Left ventricular and myocardial function in mice expressing constitutively pseudophosphorylated cardiac troponin I. Circ Res. 2009;105(12):1232-1239.

36. Murphy AM, et al. Transgenic mouse model of stunned myocardium. Science. 2000;287(5452):488-491.

37. Chen HJ, Chen YC, Hsiao CF, Chen PF. Mass spectrometric analysis of glyoxal and methylglyoxal-induced modifications in human hemoglobin from poorly controlled type 2 diabetes mellitus patients. Chem Res Toxicol. 2015;28(12):2377-2389.

38. Prochniewicz E, Thomas DD. Site-specific mutations in the myosin binding sites of actin affect structural transitions that control myosin binding. Biochemistry. 2001;40(46):13933-13940.

39. Jenkins MJ, et al. Myosin heads are displaced from actin filaments in the in situ beating rat heart in early diabetes. Biophys $J$. 2013;104(5):1065-1072.

40. Gordon AM, Homsher E, Regnier M. Regulation of contraction in striated muscle. Physiol Rev. 2000;80(2):853-924

41. Rynkiewicz MJ, et al. Tropomyosin must interact weakly with actin to effectively regulate thin filament function. Biophys $J$. 2017;113(11):2444-2451.

42. Viswanathan MC, et al. Distortion of the actin A-triad results in contractile disinhibition and cardiomyopathy. Cell Rep. 2017;20(11):2612-2625.

43. Lorenz M, Holmes KC. The actin-myosin interface. Proc Natl Acad Sci USA. 2010;107(28):12529-12534

44. Holmes KC, Schröder RR, Sweeney HL, Houdusse A. The structure of the rigor complex and its implications for the power stroke. Philos Trans R Soc Lond, B, Biol Sci. 2004;359(1452):1819-1828.

45. Behrmann E, Müller M, Penczek PA, Mannherz HG, Manstein DJ, Raunser S. Structure of the rigor actin-tropomyosin-myosin complex. Cell. 2012;150(2):327-338.

46. Ueda K, Kimura-Sakiyama C, Aihara T, Miki M, Arata T. Calcium-dependent interaction sites of tropomyosin on reconstituted muscle thin filaments with bound Myosin heads as studied by site-directed spin-labeling. Biophys J. 2013;105(10):2366-2373.

47. Kuang SQ, et al. Identification of a novel missense mutation in the cardiac beta-myosin heavy chain gene in a Chinese patient with sporadic hypertrophic cardiomyopathy. J Mol Cell Cardiol. 1996;28(9):1879-1883.

48. Hershberger RE, Norton N, Morales A, Li D, Siegfried JD, Gonzalez-Quintana J. Coding sequence rare variants identified in MYBPC3, MYH6, TPM1, TNNC1, and TNNI3 from 312 patients with familial or idiopathic dilated cardiomyopathy. Circ Cardiovasc Genet. 2010;3(2):155-161.

49. Locher MR, Razumova MV, Stelzer JE, Norman HS, Patel JR, Moss RL. Determination of rate constants for turnover of myosin isoforms in rat myocardium: implications for in vivo contractile kinetics. Am J Physiol Heart Circ Physiol. 2009;297(1):H247-H256.

50. Beisswenger PJ, Howell SK, Touchette AD, Lal S, Szwergold BS. Metformin reduces systemic methylglyoxal levels in type 2 diabetes. Diabetes. 1999;48(1):198-202.

51. Dhar A, Desai K, Liu J, Wu L. Methylglyoxal, protein binding and biological samples: are we getting the true measure? J Chromatogr B Analyt Technol Biomed Life Sci. 2009;877(11-12):1093-1100.

52. Rabbani N, Thornalley PJ. Glyoxalase in diabetes, obesity and related disorders. Semin Cell Dev Biol. 2011;22(3):309-317.

53. Talior-Volodarsky I, Connelly KA, Arora PD, Gullberg D, McCulloch CA. $\alpha 11$ integrin stimulates myofibroblast differentiation in diabetic cardiomyopathy. Cardiovasc Res. 2012;96(2):265-275.

54. Wang XL, et al. Methylglyoxal increases cardiomyocyte ischemia-reperfusion injury via glycative inhibition of thioredoxin activity. Am J Physiol Endocrinol Metab. 2010;299(2):E207-E214.

55. Willis MS, Schisler JC, Portbury AL, Patterson C. Build it up-tear it down: protein quality control in the cardiac sarcomere. Cardiovasc Res. 2009;81(3):439-448.

56. LeWinter MM, et al. Abundance, localization, and functional correlates of the advanced glycation end-product carboxymethyl lysine in human myocardium. Physiol Rep. 2017;5(20):e13462.

57. Flagg TP, et al. $\mathrm{Ca}^{2+}$-independent alterations in diastolic sarcomere length and relaxation kinetics in a mouse model of lipotoxic diabetic cardiomyopathy. Circ Res. 2009;104(1):95-103.

58. Kirk JA, et al. Cardiac resynchronization sensitizes the sarcomere to calcium by reactivating GSK-3ß. J Clin Invest. 2014;124(1):129-138.

59. Kirk JA, et al. Pacemaker-induced transient asynchrony suppresses heart failure progression. Sci Transl Med. 2015;7(319):319ra207.

60. Crisóstomo J, et al. Methylglyoxal chronic administration promotes diabetes-like cardiac ischaemia disease in Wistar normal rats. Nutr Metab Cardiovasc Dis. 2013;23(12):1223-1230.

61. Kass DA, et al. Improved arterial compliance by a novel advanced glycation end-product crosslink breaker. Circulation. 
2001;104(13):1464-1470.

62. Chandra KP, et al. Phase I clinical studies of the advanced glycation end-product (AGE)-breaker TRC4186: safety, tolerability and pharmacokinetics in healthy subjects. Clin Drug Investig. 2009;29(9):559-575.

63. Hajjar RJ, Schmidt U, Helm P, Gwathmey JK. Ca ${ }^{++}$sensitizers impair cardiac relaxation in failing human myocardium. $J$ Pharmacol Exp Ther. 1997;280(1):247-254.

64. Papadaki M, Vikhorev PG, Marston SB, Messer AE. Uncoupling of myofilament $\mathrm{Ca}^{2+}$ sensitivity from troponin I phosphorylation by mutations can be reversed by epigallocatechin-3-gallate. Cardiovasc Res. 2015;108(1):99-110.

65. Mun JY, et al. Myosin-binding protein C displaces tropomyosin to activate cardiac thin filaments and governs their speed by an independent mechanism. Proc Natl Acad Sci USA. 2014;111(6):2170-2175.

66. Patton C, Thompson S, Epel D. Some precautions in using chelators to buffer metals in biological solutions. Cell Calcium. 2004;35(5):427-431. 